defect by limiting the responsiveness toward any given antigen to only some 'classes' of $\mathrm{T}$ cell receptors, it stimulates not only further experimental protocols in $\mathbf{T}-\mathbf{B}$ collaboration but in several other systems. The importance of the Munro-Taussig work, with its demonstration of antigen specificity of the T-cell factor, the individual Ir control of $T$ cell factor and $B$ cell receptor for that factor, thus lies not only in the advance this provides for cellular immunology but also in the broader applicability of the model for cell cooperation which can now be studied at the molecular level.

In addition the two MHC Ir gene control of immune responsiveness is exciting to the geneticist. The $\mathbf{M H C}$ has often been referred to as a supergene as described by Fisher, that is, a group of linked genes that govern biologically interactive processes. During the past few years we have seen the elucidation of at least one such interacting system within the MHC where different antigens controlled by separate $\mathrm{MHC}$ genes (the SD and LD determinants) differentially activate two separate populations of interacting $\mathrm{T}$ cells ( $\mathrm{T}-\mathrm{T}$ collaboration); an interaction which leads to the effective development of cytotoxic $\mathrm{T}$ lymphocytes. Munro and Taussig in fact speculate that their model for $T-B$ collaboration may apply to $\mathrm{T}-\mathrm{T}$ interactions. It inay be that the antigens to which the separate $\mathrm{T}$ cell populations respond can also function as receptors (which may really be their major role) and that an even closer parallel than that envisioned by some will emerge between the LD-SD interaction and the T-B collaborating system.

Thus whereas many questions of key import remain (such as the possible participation of the variable part of the immunoglobulin molecule as a part of the $T$ cell receptor, the question whether there are classes of $T$ cell receptors which explain the specificity restrictions, and many others) the results and models generated by this group and others working in the same area are enormously exciting and provide a focus for the bringing together of several diverse experimental systems related to $\mathrm{MHC}$ genes.

\title{
New view of magnetic changes
}

\section{from Peter J. Smith}

THE magnetic field at the Earth's surface generally deviates from the north-south dinection (declination), certain components of the field drift slowly westwards (secular variation), and from time to time the main field changes its orientation by $180^{\circ}$ (field reversal). But although these phenomena are observable, dinectly or indirectly, their causes remain obscure. Declination, for example, is generally explained in terms of nondipole components; but insofar as the origins of the non-dipole fields are uncertain, this merely shifts the obscurity one stage further along. And although it is usual to invoke eddy currents near the edge of the core, field perturbations which trigger greater changes in a homo geneous dynamo, and so on, such 'explanations' are generally intractable to rigorous analysis.

In shanp contrast, Steenbeck and Helmis (Geophys. J., 41, 237; 1975) have now put forward-tentatively $\rightarrow$ a quite different hypothesis which is not only amenable to mathematical treatment but is also able to link declination, secular variation and field reversal within a common theoretical development. The theory relates strictly to a simple model of a particular form of gyroscope-a rigid body free to rotate within a rotating hollow sphere and weakly coupled to the sphere by isotropic friction. Steenbeck and Helmis propose, however, that the analysis may also be applicable to the Earth, with the solid core representing the rigid body of the theory.

In Earth terms, the theory predicts that varying irregularities in the magnetic field are a necessary consequence of a slow rotation of the solid core (gynoscope)-a rotation which is both possible and likely. The interaction between field and core anises basically because, being electrically conducting, the core should be penetrated by a part of the field which it then carries along. The axis of the core's rotation will lie in the Earth's equatorial plane and will notate within it, thereby giving a westward drift of varying velocity (although eastward drift could also arise under certain circumstances). But the rotational state producing the drift ultimately becomes unstable; and the solid core then tips over relatively rapidly, possibly initiating a total field reversal.

There are several problems, chief of which is that mass movements in the fluid outer core will lead to friction which is neither weak nor isotropic. But although this may alter the quantitative argument, it should not invalidate the principle.

\section{Protein folding pathfinders}

from Barry Robson

TRYING to discover how a globular protein folds up into its unique, biologically active conformation is not a task for the faint-hearted. The conformation of a protein is determined by hundreds of rotatable bonds, and hence its conformational energy can be described mathematically as a continuous surface with hundreds of dimensions. Through the hills and valleys of this conformational energy surface the molecule must weave its way in search of that single valley which represents the stable, biologically active conformation. A number of recent publications, some relating to experimental observations on folding proteins, and some to computer simulations of the folding process, give considerable insight into the most challenging and contentious aspects of the problem.

Pancreatic trypsin inhibitor is a relatively small protein of known threedimensional structure and continues to be a popular model system. Creighton (J. molec. Biol., 95, 167; 1975) continues his experimental investigation of the folding of this molecule, again forcing the formation of disulphide bridges at selected intervals of time in order to covalently cross-link the backbone chain of the molecule and so trap intermediate conformational species. In this paper he directs his attention to the analysis of those intermediate species which have two such links, and observes that the various elements of the biologically active conformation must be assembled in a definite sequence. His findings therefore support the increasingly held belief that there is a unique and obligatory pathway for folding, and that the biologically active conformation is marked not so much by its own stability as the stability of the pathway leading to it.

Creighton's emphasis on a specific folding pathway seems at first glance inconsistent with his additional discovery that the protein in the early stages of folding has considerable conformational freedom in which many different conformations are built up. However, the initial population of the disordered, fully unfolded state contains an enormous variety of conformations which must be funnelled together into the first obligatory step at the start of the pathway proper. The initial intermediates therefore resemble the routes taken by the participants on their way to the official meeting place at the start of a sponsored walk.

Events at the beginning of the folding pathway have also been recently examined in ribonuclease A. Garel and Baldwin (J. molec. Biol., 94, 611; 1975) 